\title{
GLOBAL SOLUTIONS FOR A PROBLEM MODELING THE DYNAMICS OF A SYSTEM OF SELF-GRAVITATING BROWNIAN PARTICLES
}

\author{
CAROLE ROSIER \\ Laboratoire de mathématiques pures et appliquées "Joseph Liouville", Université du Littoral \\ 50 rue F. Buisson, B.P. 699, 62228 Calais Cedex, France \\ E-mail:rosier@lmpa.univ-littoral.fr \\ LIONEL ROSIER \\ Institut Elie Cartan, Université Henri Poincaré - Nancy 1 \\ B.P. 239, 54506 Vandoeuvre-lès-Nancy Cedex, France \\ E-mail: rosier@iecn.u-nancy.fr
}

Abstract. Results on the global existence and uniqueness of variational solutions to an ellipticparabolic problem occurring in statistical mechanics are provided.

1. Introduction. Let $B$ denote the open ball in $\mathbb{R}^{3}$ centered at the origin and with radius 1 . We consider the parabolic-elliptic system

$$
\begin{gathered}
n_{\tau}=\nabla \cdot(\theta(\tau) \nabla n+n \nabla \psi) \quad \text { in } B \times \mathbb{R}^{+}, \\
\Delta \psi=4 \pi n \quad \text { in } B \times \mathbb{R}^{+},
\end{gathered}
$$

with the boundary conditions

$$
\begin{gathered}
(\theta(\tau) \nabla n+n \nabla \psi) \cdot \nu=0 \text { on } \partial B \times \mathbb{R}^{+} \\
\psi=0 \text { on } \partial B \times \mathbb{R}^{+}
\end{gathered}
$$

and the initial condition

$$
n_{\left.\right|_{\tau=0}}=n_{0},
$$

where $\int_{B} n_{0}(x) d x=1$ and $n_{0}(x) \geq 0$ for all $x \in B$. The term $\theta(\tau)$ is given at each instant

2000 Mathematics Subject Classification: 35K60, 35A07, 35B40, 82C21.

Key words and phrases: nonlocal nonlinear parabolic equation, energy conservation, global solution.

The paper is in final form and no version of it will be published elsewhere. 
by the energy conservation

$$
E=\kappa \theta(\tau)+\int_{B} n \psi d x
$$

where $E \in \mathbb{R}$ and $\kappa>0$ are given parameters. The system (1.1)-(1.6) may serve as a simplified model for the large-scale dynamics of collisionless stellar systems undergoing violent relaxation [9]. It describes also the evolution of density and temperature of a self-attracting cluster of Brownian particles in a ball of $\mathbb{R}^{3}$ in the limit of infinite thermal conductivity (see e.g. [1], [4] and [7]). Here, $\tau>0$ is time, $x$ is the spatial variable, $n$ stands for the density, $\psi$ is the gravitational potential (defined by (1.2), (1.4)), $E$ is the total energy, which is assumed to be constant, and $\theta$ is the (uniform) temperature. The local well-posedness of (1.1)-(1.6) has been investigated in [15] and [10]. Explicit solutions describing the pre-collapse and post-collapse dynamics have been obtained in $[7,8,17,18]$ for a spherically symmetric distribution of matter.

In this note we will focus on radially symmetric solutions, that is, of the form $n(x, \tau)=$ $n(r, \tau)$ where $r=|x|=\left(x_{1}^{2}+x_{2}^{2}+x_{3}^{2}\right)^{\frac{1}{2}}$. It will be assumed throughout that the initial condition $n_{0}$ is radially symmetric. As it has already been noticed in [3], [10] and [17] (1.1)-(1.2) may be combined into a single equation for the accumulated mass $Q(r, \tau):=$ $\int_{B_{r}(0)} n(x, \tau) d x=4 \pi \int_{0}^{r} n(\rho, \tau) \rho^{2} d \rho$, namely

$$
Q_{\tau}=\theta\left(Q_{r r}-\frac{2}{r} Q_{r}\right)+\frac{1}{r^{2}} Q Q_{r} .
$$

To drop the (variable) coefficient $\theta$ in front of the diffusion term $Q_{r r}$, we perform a change of time scale. We set $t=\int_{0}^{\tau} \theta(s) d s$. Then $Q_{\tau}=Q_{t} \frac{d t}{d \tau}=Q_{t} \theta(\tau)$, hence (1.7) is transformed into

$$
Q_{t}=Q_{r r}-\frac{2}{r} Q_{r}+\frac{1}{\theta r^{2}} Q Q_{r}
$$

The energy relation (1.6) becomes

$$
E=\kappa \theta-\int_{0}^{1} \frac{Q^{2}}{r^{2}} d r
$$

Finally, (1.7) is supplemented with the boundary conditions

$$
Q(0, t)=0, Q(1, t)=1,
$$

and the initial condition

$$
Q(r, 0)=Q_{0}(r)=4 \pi \int_{0}^{r} n_{0}(\rho) \rho^{2} d \rho .
$$

REMARK 1. We shall give later conditions ensuring that for all $t \geq 0$

$$
0<\theta_{1} \leq \theta(t) \leq \theta_{2}<+\infty
$$

for some constants $\theta_{1}, \theta_{2}$. As $d \tau / d t=1 / \theta(t)$, this will imply that $\tau$ ranges over $\mathbb{R}^{+}$when $t$ ranges over $\mathbb{R}^{+}$.

Blow-up results and global existence results for the problem (1.8)-(1.11) are provided in [6], [10]. They mainly rest upon some nice results obtained in [5] when the temperature is a given function and the energy relation (1.9) is removed. In [10, Thm. 1.2] it is proved 
that (1.8)-(1.11) has a global solution whenever there exists a constant $B>0$ such that

$$
Q(r)=\left\|n_{0}\right\|_{L^{1}\left(B_{r}(0)\right)} \leq(1+B) \frac{r^{3}}{r^{2}+B} \quad \text { for } r \in[0,1] \quad \text { and } \quad \theta_{0} \geq \frac{3}{2} \frac{1+B}{\lambda}
$$

where $\lambda=\lambda\left(\kappa, n_{0}\right)>0$. The assumption concerning $n_{0}$ in (1.13) amounts to saying that $n_{0}$ is bounded near the origin. However, there exist unbounded solutions of (1.1)(1.6) for which the temperature remains bounded. One of the most popular examples is provided by the Chandrasekhar stationary solution $Q_{s}(r)=r, \theta_{s}=\frac{1}{2}$ (hence $n_{0}(x)=$ Const. $|x|^{-2}$ ). One goal of this paper is to show that the global well-posedness (together with the boundedness of the temperature) may be asserted for initial data of this kind.

The paper is organized as follows. The statements of the main results are given in Section 2. The proof of one of the results (namely, Theorem 3) is sketched in Section 3.

2. Main results. To state the main results in this paper, we need to introduce a few notations. For any $k, p \in \mathbb{N}$, we let $H_{r^{p} d r}^{k}(0,1)$ denote the space of measurable functions $u:(0,1) \rightarrow \mathbb{R}$ such that

$$
\|u\|_{H_{r p d r}^{k}(0,1)}^{2}:=\int_{0}^{1}\left(u^{2}+\cdots+\left(u^{(k)}\right)^{2}\right) r^{p} d r<+\infty .
$$

When $k=0$, we write $L_{r^{p} d r}^{2}(0,1)$ instead of $H_{r^{p} d r}^{0}(0,1)$. The space $V:=\left\{u \in H_{r^{2} d r}^{1}(0,1)\right.$, $u(1)=0\}$ will be endowed with the Hilbertian norm $\|u\|_{V}^{2}=\int_{0}^{1}\left(\frac{d u}{d r}\right)^{2} r^{2} d r$. We identify the Hilbert space $L^{2}(0,1)$ (resp., $\left.L_{r^{2} d r}^{2}(0,1)\right)$ with its dual space, so that we have the diagram

$$
H_{0}^{1}(0,1) \subset L^{2}(0,1)=L^{2}(0,1)^{\prime} \subset H^{-1}(0,1) \quad\left(\text { resp., } V \subset L_{r^{2} d r}^{2}(0,1)=L_{r^{2} d r}^{2}(0,1)^{\prime} \subset V^{\prime}\right) .
$$

Finally, we introduce the spaces

$$
\begin{aligned}
& W(0, T):=\left\{u \in L^{2}\left(0, T, H_{0}^{1}(0,1)\right), u_{t} \in L^{2}\left(0,1, H^{-1}(0,1)\right)\right\} \subset C\left([0, T], L^{2}(0,1)\right), \\
& \widetilde{W}(0, T):=\left\{u \in L^{2}(0, T, V), u_{t} \in L^{2}\left(0,1, V^{\prime}\right)\right\} \subset C\left([0, T], L_{r^{2} d r}^{2}(0,1)\right) \cap L^{2}\left(0, T, L^{2}(0,1)\right) .
\end{aligned}
$$

Definition 1 . Let $Q:[0,1] \times \mathbb{R}^{+} \rightarrow[0,1]$ and $\theta: \mathbb{R}^{+} \rightarrow(0,+\infty]$ be two functions, $Q(., t)$ being nondecreasing for a.e. $t \in \mathbb{R}^{+}$. We shall say that $(Q, \theta)$ is

- a weak solution of (1.8)-(1.11) if $Q-r \in W(0, T)$ for any $T>0, \theta \in C\left(\mathbb{R}^{+},(0,+\infty)\right)$, (1.9) holds true for all $t \geq 0$, and for any $T>0$

$$
\begin{aligned}
\int_{0}^{T}\left\langle Q_{t}, v\right\rangle_{H^{-1}, H_{0}^{1}} d r d t & +\int_{0}^{T} \int_{0}^{1} Q_{r} v_{r} d r d t+2 \int_{0}^{T} \int_{0}^{1} Q_{r} \frac{v}{r} d r d t \\
& -\int_{0}^{T} \frac{1}{\theta} \int_{0}^{1} Q Q_{r} \frac{v}{r^{2}} d r d t=0 \quad \forall v \in L^{2}\left(0, T, H_{0}^{1}(0,1)\right)
\end{aligned}
$$

- a very weak solution of (1.8)-(1.11) if $Q-r \in \widetilde{W}(0, T) \cap L^{2}\left(0, T, H_{r d r}^{1}(0,1)\right)$ for any $T>0, \theta^{-1} \in L^{\infty}\left(\mathbb{R}^{+}\right)$and $(Q, 1 / \theta)$ may be obtained as a "weak limit" of a sequence $\left(Q^{k}, 1 / \theta^{k}\right)$ of solutions to the approximate problems (3.10)-(3.13). By a weak limit, we 
mean that

$$
\begin{aligned}
\frac{1}{\theta^{k}} \rightarrow \frac{1}{\theta} & \text { in } L^{\infty}(0, T) \text {-weak } \\
Q^{k}-r \rightarrow Q-r & \text { in } L^{2}(0, T, V), \\
Q_{t}^{k} \rightarrow Q_{t} & \text { in } L^{2}\left(0, T, V^{\prime}\right)
\end{aligned}
$$

as $k \rightarrow+\infty$, for any $T>0$.

Let us point out that a very weak solution satisfies the following variational formulation:

$$
\begin{aligned}
\int_{0}^{T}\left\langle Q_{t}, v\right\rangle_{V^{\prime}, V} d t & +\int_{0}^{T} \int_{0}^{1} Q_{r} v_{r} r^{2} d r d t+4 \int_{0}^{T} \int_{0}^{1} Q_{r} v r d r d t \\
+ & \frac{1}{2} \int_{0}^{T} \frac{1}{\theta(t)}\left(\int_{0}^{1} Q^{2} v_{r} d r\right) d t=0 \quad \forall v \in C_{0}^{\infty}((0,1) \times(0, T)) .
\end{aligned}
$$

The main drawback of this notion is that the validity of (1.9) is uncertain. On the other hand, the boundary condition $Q(0, t)=0$ may fail to be true. This is the case for the stationary very weak solution $(Q, \theta)=(1,+\infty)$ (corresponding to the density $n_{0}=\delta_{x=0}$ ).

The first result of the paper is concerned with the global existence of weak solutions.

TheOREM 1. Let $Q_{0} \in H^{1}(0,1)$ be a nondecreasing function such that $Q_{0}(0)=0$, $Q_{0}(1)=1$, and let $\lambda=\exp \left(-\frac{2}{\kappa} \int_{0}^{1} \frac{d Q_{0}}{d r} \log \left(r^{-2} \frac{d Q_{0}}{d r}\right) d r\right)$. Assume that there exists a constant $C \geq 1$ such that

$$
Q_{0}(r) \leq C r \quad \forall r \in[0,1]
$$

and

$$
\theta_{0}:=\kappa^{-1}\left(E+\int_{0}^{1}\left(\frac{Q_{0}(r)}{r}\right)^{2} d r\right)>\frac{C}{2 \lambda} .
$$

Then (1.8)-(1.11) has a weak solution $(Q, \theta)$ defined on $\mathbb{R}^{+}$and fulfilling

$$
0 \leq Q(r, t) \leq \min (C r, 1) \quad \forall r \in[0,1] \times \mathbb{R}^{+}
$$

and

$$
0<\lambda \theta_{0} \leq \theta(t) \leq \theta_{M}<+\infty \quad \forall t \in \mathbb{R}^{+},
$$

where $\theta_{M}:=\kappa^{-1}\left(E+C^{2}\right)=\theta_{0}+\kappa^{-1}\left(C^{2}-\int_{0}^{1}\left(\frac{Q_{0}(r)}{r}\right)^{2} d r\right)$.

REMARK 2.

1. The assumption (2.6) is satisfied whenever the Morrey norm of exponent $3 / 2$ of the initial density $n_{0}$ is finite, that is,

$$
\left\|n_{0}\right\|_{M^{\frac{3}{2}(B)}}:=\sup _{x \in B, 0 \leq r \leq 1} r^{-1}\left\|n_{0}\right\|_{L^{1}\left(B \cap B_{r}(x)\right)}<\infty .
$$

This is e.g. the case when $n_{0} \in L^{p}(B)$ with $p \geq \frac{3}{2}$, or when $n_{0}(x) \leq$ Const. $|x|^{-2}$.

2. The temperature remains bounded even if the density is of order $|x|^{-2}$ near the origin.

3. Theorem 1 can be applied to initial conditions close to the Chandrasekhar stationary solution in the limit $\kappa \rightarrow+\infty$.

The second main result in this paper is concerned with the uniqueness of radially symmetric solutions. 
TheOREM 2. Let $Q_{0}, \theta_{0}$ be as in Theorem 1. Assume further that

$$
\theta_{0} \geq \frac{2 C^{2}}{\kappa \lambda\left(\sqrt{1+\frac{8 C}{5 \kappa}}-1\right)} .
$$

Then the weak solution given in Theorem 1 is unique.

The proofs of Theorem 1 and Theorem 2 will be given in [16].

The third result, whose proof is sketched here, is concerned with the existence of very weak solutions.

THEOREM 3. Let $Q_{0} \in H^{1}(0,1)$ be a nondecreasing function with $Q_{0}(0)=0, Q_{0}(1)=1$ and such that $\theta_{0}:=\kappa^{-1}\left(E+\int_{0}^{1} Q_{0}^{2} r^{-2} d r\right)>0$. Then (1.8)-(1.11) has a very weak solution $(Q, \theta)$ defined on $\mathbb{R}^{+}$.

\section{Proof of Theorem 3}

STEP 1. Construction of an approximate solution. Let $\left(\varepsilon^{k}\right)_{k \geq 0}$ denote a sequence of positive real numbers such that $\varepsilon^{k} \searrow 0$ as $k \rightarrow \infty$. We consider the following approximate problem: find a function $Q^{k}:[0,1] \times \mathbb{R}^{+} \rightarrow \mathbb{R}$ fulfilling

$$
\begin{gathered}
Q_{t}^{k}=Q_{r r}^{k}-\frac{2}{r+\varepsilon^{k}} Q_{r}^{k}+\frac{1}{\left(\left|\theta^{k}\right|+\varepsilon^{k}\right)\left(r+\varepsilon^{k}\right)^{2}} T\left(Q^{k}\right) Q_{r}^{k} \quad \text { on }[0,1] \times \mathbb{R}^{+} \\
\theta^{k}=\kappa^{-1}\left(E+\int_{0}^{1}\left(\frac{T\left(Q^{k}\right)}{r+\varepsilon^{k}}\right)^{2} d r\right) \\
Q^{k}(0, t)=0, \quad Q^{k}(1, t)=1 . \\
Q^{k}(r, 0)=Q_{0}(r) .
\end{gathered}
$$

In (3.1) the function $T: \mathbb{R} \rightarrow \mathbb{R}$ is defined by

$$
T(s)= \begin{cases}0 & \text { if } s \leq 0 \\ s & \text { if } 0 \leq s \leq 1 \\ 1 & \text { if } s \geq 1\end{cases}
$$

The global well-posedness of (3.1)-(3.4) is stated in the following result, whose proof is left to the reader.

Proposition 1. Let $Q_{0} \in H^{1}(0,1)$ be such that $Q_{0}(0)=0, Q_{0}(1)=1, Q_{0}^{\prime} \geq 0$ and $\theta_{0}:=$ $\kappa^{-1}\left(E+\int_{0}^{1} Q_{0}^{2} r^{-2} d r\right)>0$. Then there exists a unique solution $Q^{k}:[0,1] \times \mathbb{R}^{+} \rightarrow \mathbb{R}$ of (3.1)-(3.4) in the class $C\left([0,+\infty), H^{1}(0,1)\right) \cap C\left((0,+\infty), H^{2}(0,1)\right) \cap C^{1}\left((0,+\infty), L^{2}(0,1)\right)$.

STEP 2. Boundedness of the approximate solution. To be physically relevant, the density $n(r, t)$ has to remain nonnegative, which amounts to saying that the corresponding accumulated mass $Q$ is at each instant a nondecreasing function of $r$. That property turns out to be satisfied for the solution $Q^{k}$ of the approximate system (3.1)-(3.4).

Proposition 2 ([16]). Let $Q^{k}$ be the solution of (3.1)-(3.4) given by Proposition 1. Then

$$
\frac{d Q^{k}}{d r}(r, t) \geq 0 \quad \forall(r, t) \in[0,1] \times(0,+\infty) .
$$


As a consequence, we have that

$$
0 \leq Q^{k}(r, t) \leq 1 \quad \forall(r, t) \in[0,1] \times \mathbb{R}^{+} .
$$

Using (3.7), we may drop the function $T$ in (3.1)-(3.2).

Step 3. Positivity of the temperature. We claim that $\theta^{k}$ remains bounded from below by a positive constant independent of $k$. To establish this property we introduce the Boltzmann entropy

$$
S^{k}(t):=\int_{0}^{1} Q_{r}^{k} \log \left[\frac{Q_{r}^{k}}{\left(r+\varepsilon^{k}\right)^{2}}\right] d r-\frac{\kappa}{2} \log \left|\theta^{k}+\varepsilon^{k}\right| .
$$

Notice that the integral term in (3.8) is well defined as $Q^{k} \in C\left(\mathbb{R}^{+}, H^{1}(0,1)\right)$. This functional actually plays the role of a Liapunov function for (3.1)-(3.4).

Proposition 3 ([16]). The function $S^{k}$ is non-increasing.

After some computations we arrive to the following result.

COROllary 1. There exists some integer $k_{0} \geq 0$ such that for any $k \geq k_{0}$ and any $t \in[0,+\infty)$,

$$
\theta^{k}(t) \geq\left(1+\varepsilon^{k}\right)^{-\frac{4}{\kappa}} \exp \left(-\frac{2}{\kappa} S^{k}(0)\right)-\varepsilon^{k}>0 .
$$

At this stage $Q^{k}$ solves, for any $k \geq k_{0}$, the system

$$
\begin{gathered}
Q_{t}^{k}=Q_{r r}^{k}-\frac{2}{r+\varepsilon^{k}} Q_{r}^{k}+\frac{1}{\left(\theta^{k}+\varepsilon^{k}\right)\left(r+\varepsilon^{k}\right)^{2}} Q^{k} Q_{r}^{k} \quad \text { on }[0,1] \times \mathbb{R}^{+} \\
\theta^{k}=\kappa^{-1}\left(E+\int_{0}^{1}\left(\frac{Q^{k}}{r+\varepsilon^{k}}\right)^{2} d r\right) \\
Q^{k}(0, t)=0, \quad Q^{k}(1, t)=1 . \\
Q^{k}(r, 0)=Q_{0}(r) .
\end{gathered}
$$

STEP 4. Some estimates for the approximate solution. We establish some estimates needed to pass to the limit in (3.10)-(3.13).

Proposition 4.

$$
\begin{gathered}
\sup _{k \geq 0}\left\|Q^{k}-r\right\|_{L^{2}(0, T, V)} \leq \sup _{k \geq 0}\left\|Q^{k}-r\right\|_{L^{2}\left(0, T, H_{r d r}^{1}(0,1)\right)}<+\infty, \\
\sup _{k \geq 0}\left\|Q_{t}^{k}\right\|_{L^{2}\left(0, T, V^{\prime}\right)}<+\infty .
\end{gathered}
$$

Proof. In what follows, the number $K$ will denote a positive constant, which may vary from line to line, and which depends only on $T, \kappa, E$ and $Q_{0}$. We consider the function $M^{k}=Q^{k}-r$ which fulfills

$$
\begin{gathered}
M_{t}^{k}=M_{r r}^{k}-\frac{2}{r+\varepsilon^{k}}\left(M_{r}^{k}+1\right)+\frac{1}{\left(\theta^{k}+\varepsilon^{k}\right)\left(r+\varepsilon^{k}\right)^{2}}\left(M^{k}+r\right)\left(M_{r}^{k}+1\right) \\
M^{k}(0, t)=M^{k}(1, t)=0 .
\end{gathered}
$$


Scaling in (3.16) by $M^{k} r$ and integrating by parts, we obtain for all $t>0$

$$
\begin{aligned}
\int_{0}^{1} M_{t}^{k} M^{k} r d r= & \int_{0}^{1} M_{r r}^{k} M^{k} r d r-2 \int_{0}^{1}\left(M_{r}^{k}+1\right) M^{k} \frac{r}{r+\varepsilon^{k}} d r \\
& +\frac{1}{\left(\theta^{k}+\varepsilon^{k}\right)} \int_{0}^{1}\left(M^{k}+r\right)\left(M_{r}^{k}+1\right) M^{k} \frac{r}{\left(r+\varepsilon^{k}\right)^{2}} d r \\
= & -\int_{0}^{1}\left(M_{r}^{k}\right)^{2} r d r-2 \int_{0}^{1}\left(M_{r}^{k}+1\right)\left(M^{k}+r\right) \frac{r}{r+\varepsilon^{k}} d r \\
& +2 \int_{0}^{1}\left(M_{r}^{k}+1\right) \frac{r^{2}}{r+\varepsilon^{k}} d r \\
& +\frac{1}{\left(\theta^{k}+\varepsilon^{k}\right)} \int_{0}^{1}\left[\left(M^{k}+r\right)^{2}-\left(M^{k}+r\right) r\right]\left(M_{r}^{k}+1\right) \frac{r}{\left(r+\varepsilon^{k}\right)^{2}} d r \\
= & -\int_{0}^{1}\left(M_{r}^{k}\right)^{2} r d r-2 \int_{0}^{1}\left(M_{r}^{k}+1\right)\left(M^{k}+r\right) \frac{r}{r+\varepsilon^{k}} d r \\
& -2 \int_{0}^{1}\left(M^{k}+r\right) \frac{r^{2}+2 r \varepsilon^{k}}{\left(r+\varepsilon^{k}\right)^{2}} d r+\frac{2}{1+\varepsilon^{k}} \\
& +\frac{1}{\theta^{k}+\varepsilon^{k}}\left\{-\int_{0}^{1} \frac{\left(M^{k}+r\right)^{3}}{3} \frac{\varepsilon^{k}-r}{\left(r+\varepsilon^{k}\right)^{3}} d r\right. \\
& \left.+\frac{1}{3} \frac{1}{\left(1+\varepsilon^{k}\right)^{2}}-\int_{0}^{1}\left(M^{k}+r\right)\left(M_{r}^{k}+1\right) \frac{r^{2}}{\left(r+\varepsilon^{k}\right)^{2}} d r\right\} .
\end{aligned}
$$

Integrating over $(\delta, T)$ and letting $\delta \rightarrow 0^{+}$, we obtain

$$
\begin{aligned}
\frac{1}{2} \int_{0}^{1}\left(M^{k}\right)^{2}(r, T) r d r+ & \int_{0}^{T} \int_{0}^{1}\left(M_{r}^{k}\right)^{2} r d r d t+2 \int_{0}^{T} \int_{0}^{1}\left(M_{r}^{k}+1\right)\left(M^{k}+r\right) \frac{r}{r+\varepsilon^{k}} d r d t \\
& +\int_{0}^{T} \frac{1}{\theta^{k}+\varepsilon^{k}} \int_{0}^{1}\left(M^{k}+r\right)\left(M_{r}^{k}+1\right) \frac{r^{2}}{\left(r+\varepsilon^{k}\right)^{2}} d r d t \\
= & \frac{1}{2} \int_{0}^{1}\left(M_{0}(r)\right)^{2} r d r-2 \int_{0}^{T} \int_{0}^{1}\left(M^{k}+r\right) \frac{r^{2}+2 r \varepsilon^{k}}{\left(r+\varepsilon^{k}\right)^{2}} d r d t+\frac{2 T}{1+\varepsilon^{k}} \\
& +\int_{0}^{T} \frac{1}{\theta^{k}+\varepsilon^{k}}\left(\frac{1}{3} \int_{0}^{1}\left(M^{k}+r\right)^{3} \frac{r-\varepsilon^{k}}{\left(r+\varepsilon^{k}\right)^{3}} d r+\frac{1}{3} \frac{1}{\left(1+\varepsilon^{k}\right)^{2}}\right) d t .
\end{aligned}
$$

As $0 \leq M^{k}+r \leq 1, M_{r}^{k}+1 \geq 0,\left|\frac{r^{2}+2 r \varepsilon^{k}}{\left(r+\varepsilon^{k}\right)^{2}}\right| \leq 2$ and

$$
\int_{0}^{1}\left(M^{k}+r\right)^{3} \frac{\left|r-\varepsilon^{k}\right|}{\left(r+\varepsilon^{k}\right)^{3}} d r \leq \int_{0}^{1}\left(\frac{M^{k}+r}{r+\varepsilon^{k}}\right)^{2} d r=\kappa \theta^{k}-E
$$

we infer that

$$
\int_{0}^{T} \int_{0}^{1}\left(M_{r}^{k}\right)^{2} r d r d t \leq K
$$

This yields (3.14). We now proceed to the proof of (3.15). Let $v \in C_{0}^{\infty}(0, T, V)$. Scaling in $(3.16)$ by $v r^{2}$ and integrating over $(0,1) \times(0, T)$, we obtain after some integrations by 
parts

$$
\begin{aligned}
\int_{0}^{T} \int_{0}^{1} M_{t}^{k} v r^{2} d r d t= & -\int_{0}^{T} \int_{0}^{1}\left(M_{r}^{k}+1\right)\left(r^{2} v_{r}+2 r v\right) d r d t-2 \int_{0}^{T} \int_{0}^{1}\left(M_{r}^{k}+1\right) v \frac{r^{2}}{r+\varepsilon^{k}} d r d t \\
& +\int_{0}^{T} \frac{1}{\theta^{k}+\varepsilon^{k}}\left(\int_{0}^{1}\left(M^{k}+r\right)\left(M_{r}^{k}+1\right) v \frac{r^{2}}{\left(r+\varepsilon^{k}\right)^{2}} d r\right) d t \\
= & I_{1}+I_{2}+I_{3} .
\end{aligned}
$$

Clearly,

$$
\begin{aligned}
\left|I_{1}\right| \leq & \left(\int_{0}^{T} \int_{0}^{1}\left(M_{r}^{k}+1\right)^{2} r^{2} d r d t\right)^{\frac{1}{2}}\left(\int_{0}^{T} \int_{0}^{1}\left(v_{r}\right)^{2} r^{2} d r d t\right)^{\frac{1}{2}} \\
& +2 \int_{0}^{T}\left(\int_{0}^{1}\left|M_{r}^{k}+1\right| d r\right)\|r v\|_{L^{\infty}(0,1)} d t \\
\leq & K\|v\|_{L^{2}(0, T, V)} .
\end{aligned}
$$

Indeed, $\int_{0}^{1}\left|M_{r}^{k}+1\right| d r=\int_{0}^{1} Q_{r}^{k} d r=1$, and it may be proved that $\|r v\|_{L^{\infty}(0,1)} \leq K\|v\|_{V}$. A similar computation gives

$$
\left|I_{2}\right| \leq\left. K|| v\right|_{L^{2}(0, T, V)}
$$

Integrating by parts in $I_{3}$, we obtain

$$
\begin{aligned}
I_{3}= & -\frac{1}{2} \int_{0}^{T} \frac{1}{\theta^{k}+\varepsilon^{k}}\left(\int_{0}^{1}\left(M^{k}+r\right)^{2} v_{r} \frac{r^{2}}{\left(r+\varepsilon^{k}\right)^{2}} d r\right) d t \\
& -\int_{0}^{T} \frac{1}{\theta^{k}+\varepsilon^{k}}\left(\int_{0}^{1}\left(M^{k}+r\right)^{2} v \frac{r \varepsilon^{k}}{\left(r+\varepsilon^{k}\right)^{3}} d r\right) d t \\
= & J_{1}+J_{2} .
\end{aligned}
$$

Then, by the Cauchy-Schwarz inequality,

$$
\begin{aligned}
\left|J_{1}\right| & \leq \frac{1}{2} \int_{0}^{T} \frac{1}{\theta^{k}+\varepsilon^{k}}\left(\int_{0}^{1}\left(M^{k}+r\right)^{4} \frac{r^{2}}{\left(r+\varepsilon^{k}\right)^{4}} d r\right)^{\frac{1}{2}}\|v\|_{V} d t \\
& \leq \frac{1}{2}\left(\int_{0}^{T} \frac{1}{\left(\theta^{k}+\varepsilon^{k}\right)^{2}}\left(\int_{0}^{1}\left(\frac{M^{k}+r}{r+\varepsilon^{k}}\right)^{2} d r\right) d t\right)^{\frac{1}{2}}\|v\|_{L^{2}(0, T, V)} \\
& \leq \frac{1}{2}\left(\int_{0}^{T} \frac{\kappa \theta^{k}+|E|}{\left(\theta^{k}+\varepsilon^{k}\right)^{2}} d t\right)^{\frac{1}{2}}\|v\|_{L^{2}(0, T, V)} \\
& \leq K\|v\|_{L^{2}(0, T, V)}
\end{aligned}
$$

and

$$
\begin{aligned}
\left|J_{2}\right| & \leq \int_{0}^{T} \frac{1}{\theta^{k}+\varepsilon^{k}}\left(\int_{0}^{1} \frac{M^{k}+r}{r+\varepsilon^{k}}|v| d r\right) d t \\
& \leq \int_{0}^{T} \frac{1}{\theta^{k}+\varepsilon^{k}}\left(\int_{0}^{1}\left(\frac{M^{k}+r}{r+\varepsilon^{k}}\right)^{2} d r\right)^{\frac{1}{2}}\left(\int_{0}^{1} v^{2} d r\right)^{\frac{1}{2}} d t \\
& \leq K\|v\|_{L^{2}\left(0, T, L^{2}(0,1)\right)} \\
& \leq K\|v\|_{L^{2}(0, T, V)} .
\end{aligned}
$$


Gathering (3.18)-(3.24) we obtain that for any $v \in C_{0}^{\infty}(0, T, V)$

$$
\left|\int_{0}^{T}\left\langle M_{t}^{k}, v\right\rangle_{V^{\prime}, V} d t\right| \leq K\|v\|_{L^{2}(0, T, V)},
$$

which yields (3.15).

Step 5. Passage to the limit. Let $T>0$ be given. Using (3.14), (3.15) we infer the existence of a function $M \in \widetilde{W}(0, T)$ such that, for a subsequence of $\left(M^{k}\right)$ (again denoted by $\left.\left(M^{k}\right)\right)$,

$$
\begin{array}{ll}
M^{k} \rightarrow M & \text { in } L^{2}(0, T, V) \\
M_{t}^{k} \rightarrow M_{t} & \text { in } L^{2}\left(0, T, V^{\prime}\right)
\end{array}
$$

As the first embedding in

$$
V \subset L_{r^{2} d r}^{2}(0,1) \subset V^{\prime}
$$

is compact, extracting a subsequence if needed we obtain from Aubin's lemma that

$$
M^{k} \rightarrow M \quad \text { in } L^{2}\left(0, T, L_{r^{2} d r}^{2}(0,1)\right) \quad \text { and a.e. }
$$

as $k \rightarrow+\infty$. Scaling in (3.16) by $r^{2} v$ with $v \in C_{0}^{\infty}((0,1) \times(0, T))$, we obtain after some integration by parts

$$
\begin{aligned}
& \int_{0}^{T}\left\langle M_{t}^{k}, v\right\rangle_{V^{\prime}, V} d t+\int_{0}^{T} \int_{0}^{1}\left(M_{r}^{k}+1\right)\left(r^{2} v_{r}+2 r v\right) d r d t+2 \int_{0}^{T} \int_{0}^{1}\left(M_{r}^{k}+1\right) v \frac{r^{2}}{r+\varepsilon^{k}} d r d t \\
& +\frac{1}{2} \int_{0}^{T} \frac{1}{\theta^{k}+\varepsilon^{k}}\left(\int_{0}^{1}\left(M^{k}+r\right)^{2} v_{r} \frac{r^{2}}{\left(r+\varepsilon^{k}\right)^{2}} d r\right) d t \\
& +\int_{0}^{T} \frac{1}{\theta^{k}+\varepsilon^{k}}\left(\int_{0}^{1}\left(M^{k}+r\right)^{2} v \frac{r \varepsilon^{k}}{\left(r+\varepsilon^{k}\right)^{3}} d r\right) d t \\
& =I_{1}+I_{2}+I_{3}+I_{4}+I_{5}=0 .
\end{aligned}
$$

Clearly, by (3.25)-(3.26),

$$
\begin{aligned}
I_{1}+I_{2}+I_{3} \rightarrow \int_{0}^{T}\left\langle M_{t}, v\right\rangle_{V^{\prime}, V} d t & +\int_{0}^{T} \int_{0}^{1}\left(M_{r}+1\right)\left(r^{2} v_{r}+2 r v\right) d r d t \\
& +2 \int_{0}^{T} \int_{0}^{1}\left(M_{r}+1\right) r v d r d t
\end{aligned}
$$

as $k \rightarrow \infty$. $\theta^{k}$ being bounded from below according to Corollary 1 , extracting a subsequence if needed we may assume that

$$
\frac{1}{\theta^{k}} \rightarrow \frac{1}{\theta} \quad \text { in } L^{\infty}(0, T) \text { weak }^{*}
$$

for some function $\theta: \mathbb{R} \rightarrow(0,+\infty]$ whose inverse is bounded. As $\left(M^{k}+r\right)^{2} v_{r} r^{2}(r+$ $\left.\varepsilon^{k}\right)^{-2} \rightarrow(M+r)^{2} v_{r}$ and $\left(M^{k}+r\right)^{2} v r \varepsilon^{k}\left(r+\varepsilon^{k}\right)^{-3} \rightarrow 0$ in $L^{1}((0,1) \times(0, T))$ when $k \rightarrow \infty$, we infer that

$$
I_{4}+I_{5} \rightarrow \frac{1}{2} \int_{0}^{T} \frac{1}{\theta} \int_{0}^{1}(M+r)^{2} v_{r} d r .
$$

Hence, (2.5) is satisfied. Using a diagonal process, we may assume that (3.25)-(3.28) hold true for any $T>0$. The proof of Theorem 3 is complete. 
Acknowledgments. The first author would like to thank P. Biler and P.-H. Chavanis for a stimulating discussion during the course of this work.

\section{References}

[1] P. Biler, J. Dolbeault, M. J. Esteban, P. A. Markowich and T. Nadzieja, Steady states for Streater's energy transport models of self-gravitating particles, in: Transport in Transition Regimes, N. Ben Abdallah, A. Arnold, P. Degond et al. (eds.), Springer IMA Volumes in Mathematics and Its Applications 135, Springer, 2003, 37-56.

[2] P. Biler, W. Hebisch and T. Nadzieja, The Debye system: existence and long time behaviour of solutions, Nonlinear Analysis 23 (1994), 1189-1209.

[3] P. Biler, D. Hilhorst and T. Nadzieja, The Debye system: existence nonexistence of solutions for a model of gravitational interaction of particules, II, Colloq. Math. 67 (1994), 297-308.

[4] P. Biler, A. Krzywicki and T. Nadzieja, Self-interaction of Brownian particles coupled with thermodynamic processes, Rep. Math. Phys. 42 (1998), 359-372.

[5] P. Biler and T. Nadzieja, Growth and accretion of mass in an astrophysical model II, Applicationes Math. 23 (1995), 351-361.

[6] P. Biler and T. Nadzieja, Global and exploding solutions in a model of self-gravitating systems, Rep. Math. Phys. 52 (2003), 205-225.

[7] P. H. Chavanis, C. Rosier and C. Sire, Thermodynamics of self-gravitating systems, Phys. Rev. E 66 (2002), 036105.

[8] P. H. Chavanis and C. Sire, Anomalous diffusion and collapse of self-gravitating Langevin particles in D dimensions, Phys. Rev. E 69 (2004), 016116.

[9] P. H. Chavanis, J. Sommeria and R. Robert, Statistical mechanics of two-dimensional vortices and collisionless stellar systems, Astrophys. J. 471 (1996), 385-399.

[10] C. J. van Duijn, I. A. Guerra and M. A. Peletier, Global existence conditions for a non-local problem arising in statistical mechanics, Adv. Diff. Eq. 9 (2004), 133-158.

[11] L. C. Evans, Partial Differential Equations, Graduate Studies in Mathematics 19, AMS, 1998.

[12] D. Henry, Geometric Theory of Semilinear Parabolic Equations, Lecture Notes in Math. 840, Springer, 1981.

[13] J. L. Lions and E. Magenes, Problèmes aux limites non homogènes et applications, Vol. 1, Dunod, 1968.

[14] D. Hilhorst, A nonlinear evolution problem arising in the physics of ionized gases, SIAM J. Math. Anal. 13 (1982), 16-39.

[15] C. Rosier, Problème de Cauchy pour une équation parabolique modélisant la relaxation des systèmes auto-gravitant, C. R. Acad. Sci. Paris Série I 332 (2001), 903-908.

[16] C. Rosier and L. Rosier, On the global existence of solutions for a non-local problem occurring in statistical mechanics, in preparation.

[17] C. Sire and P. H. Chavanis, Thermodynamics and collapse of self-gravitating Brownian particles in D dimensions, Phys. Rev. E 66 (2002), 0461331.

[18] C. Sire and P. H. Chavanis, Post-collapse dynamics of self-gravitating Brownian particles and bacterial populations, Phys. Rev. E 69 (2004), 066109. 